\title{
Alterstice
}

Revue internationale de la recherche interculturelle

International Journal of Intercultural Research

Revista International de la Investigacion Intercultural

\section{Communication et interculturalité : cultures et interactions interpersonnelles, de Alexander Frame}

\section{Rhéa Joanne Rocque}

Volume 3, numéro 2, 2013

URI : https://id.erudit.org/iderudit/1077525ar

DOI : https://doi.org/10.7202/1077525ar

Aller au sommaire du numéro

Éditeur(s)

Alterstice

ISSN

1923-919X (numérique)

Découvrir la revue

Citer ce compte rendu

Rocque, R. J. (2013). Compte rendu de [Communication et interculturalité :

cultures et interactions interpersonnelles, de Alexander Frame]. Alterstice, 3(2),

111-117. https://doi.org/10.7202/1077525ar d'utilisation que vous pouvez consulter en ligne.

https://apropos.erudit.org/fr/usagers/politique-dutilisation/ 


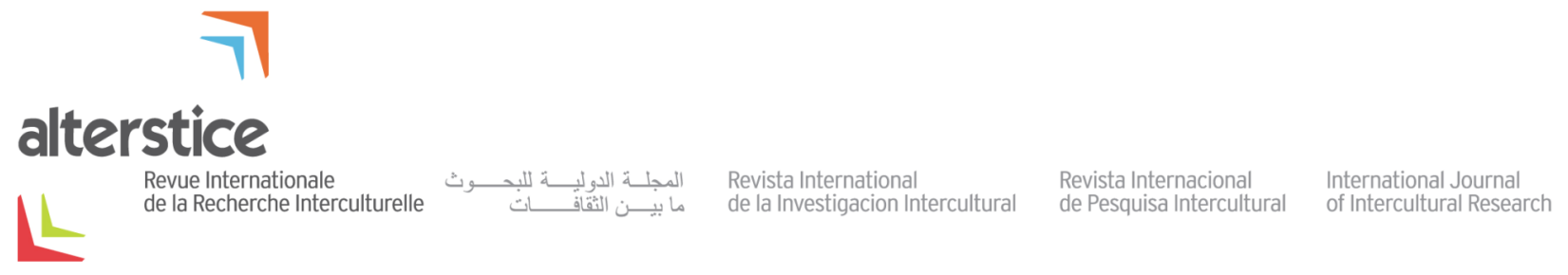

NOTE DE LECTURE

\title{
Communication et interculturalité : cultures et interactions interpersonnelles, de Alexander Frame
}

Rhéa Joanne Rocque ${ }^{1}$

\author{
Rattachement de l'auteur \\ ${ }^{1}$ École de psychologie, Université Laval, Québec, Canada
}

\section{Correspondance}

rhea.rocque.1@ulaval.ca

\section{Références de l'ouvrage :}

Frame, A. (2013). Communication et interculturalité : cultures et interactions interpersonnelles. Paris: HermèsLavoisier, $334 \mathrm{p}$.

\section{Pour citer cet article :}

Rocque, R. J. (2013). Communication et interculturalité : cultures et interactions interpersonnelles [note de lecture]. Alterstice, 3(2), 111-117.

\section{Présentation générale}

Cet ouvrage, rédigé par Alexander Frame, a pour objectif d'offrir une vue d'ensemble de quelques concepts de base pertinents au domaine de la communication interculturelle. C'est en retravaillant ces concepts que l'auteur propose un nouveau cadre théorique permettant de mieux penser la communication interculturelle. II nomme son approche sémiopragmatique. Étant donné la richesse théorique présentée et la complexité de certains concepts, ce livre cible principalement un public universitaire intéressé par les dynamiques existant entre cultures, identités et interactions, et ce, tout en adoptant la vision des sciences de l'information et de la communication. Divisé en trois parties ( 6 chapitres), il s'avère très pertinent et sera d'une grande aide à tous les penseurs, chercheurs et étudiants qui traitent de communication interculturelle.

Il importe également de souligner la définition élargie de la notion de culture à laquelle Alexander Frame adhère. La culture englobe, selon l'auteur, non seulement les cultures nationales, mais également tous les autres groupes culturels (culture organisationnelle, culture générationnelle, etc.). Cette définition élargie permet ainsi d'appliquer ce modèle à toutes situations d'interaction. 


\section{Première partie : Comprendre la communication entre étrangers}

\section{Chapitre 1 : Quels modèles pour comprendre les interactions entre étrangers?}

Il faut d'abord relever l'usage particulier que fait l'auteur du terme "étranger ». En faisant allusion à la communication entre étrangers, l'auteur considère non seulement les interactions entre individus de différentes nationalités, mais, voire au contraire, il considère que toutes interactions impliquent deux étrangers. Le terme "étranger", selon Alexander Frame, fait donc référence à toute personne qui appartient à d'autres groupes sociaux que le sien. En raison du danger de confusion, certains chercheurs et théoriciens préfèrent le terme $d^{\prime}$ « Autre».

Le premier chapitre sert à réviser les différents modèles préexistants qui servent à comprendre et à expliquer la communication. Deux courants majeurs sont présentés : les modèles anglophones et les modèles francophones, ainsi que leurs forces et leurs faiblesses lorsqu'ils sont utilisés pour étudier la communication entre étrangers.

Les modèles théoriques anglophones sont issus des domaines de recherche "cross cultural» (approches comparatives) et "d'interculturalité » (p. ex. dimension individualiste/collectiviste des cultures, voir Hofstede [1980], psychologue social). Bien que ces approches permettent de prendre conscience des différences qui existent entre cultures, elles deviennent dangereuses lorsque l'on considère ces différences comme constantes et non variables.

Par la suite, deux modèles théoriques anglophones qui cherchent à comprendre la communication interculturelle sont présentés, la Anxiety/Uncertainty Management Theory (AUM) de William Gudykunst (1995), professeur en communication, ainsi que la Communication Accomodation Theory (CAT) de Howard Giles (1973), psycholinguiste. Bien que ces théories servent à conceptualiser les interactions en contexte interculturel, elles semblent négliger l'influence de plusieurs autres facteurs sur l'efficacité de la communication ou la capacité des individus à prévoir les réactions de l'Autre.

Le chapitre se termine en passant en révision le modèle d'interactionnisme symbolique (plusieurs chercheurs et écoles ont contribué à ce modèle, notons entre autres, Blumer [1969] et Stryker [1980], sociologues), modèle francophone axé davantage sur l'individu en tant qu'agent actif cherchant à faire sens de la situation en se basant sur les normes sociales et codes préexistants, tout en agissant conformément aux normes prescrites aux rôles sociaux qu'il occupe. L'auteur note cependant que la majorité des applications de ce modèle ciblaient des individus de même groupe culturel.

Alexander Frame entreprend ensuite la tâche complexe de lier ces deux courants afin de prendre en considération à la fois les caractéristiques culturelles, en s'appuyant sur les approches comparatives, et les caractéristiques individuelles associées aux interactions, notamment les questions identitaires.

\section{Chapitre 2 : Figures de l'étranger dans les interactions}

Contrairement aux approches interactionnistes, Alexander Frame s'intéresse à la communication entre individus de différentes cultures. Il s'avère donc important d'étudier les modalités de représentations de l'étranger et comment ces dernières peuvent en retour jouer sur les relations interpersonnelles.

Dans ce deuxième chapitre, un modèle tridimensionnel de représentation de l'étranger est présenté. Les trois dimensions sont celles de la différenciation, de la particularisation et de la complexité identitaire. Les deux premières dimensions sont d'abord précisées. La représentation de l'étranger peut varier selon le degré auquel on remarque que l'étranger provient d'une culture différente de la nôtre (différenciation). Elle varie aussi selon le degré auquel on reconnaît que l'identité de l'étranger n'est pas uniquement celle de son groupe (particularisation). Le positionnement d'un étranger sur ces deux dimensions combinées débouche sur quatre profils de représentations possibles: le sosie culturel, le barbare, le stéréotype et le prototype individualisé. À titre d'exemple, si une Suédoise rencontre une Canadienne et perçoit qu'il existe une importante différence entre elles (pôle positif de la différenciation) et l'étiquette principalement de Canadienne (pôle négatif de la particularisation),

Alterstice-Revue Internationale de la Recherche Interculturelle, vol. 3, $n^{\circ} 2$ 
il s'agira de la figure du stéréotype. Si la Suédoise la perçoit plutôt comme différente des autres membres de la culture canadienne (pôle positif de la particularisation), il s'agira de la figure du prototype individualisé.

Les représentations de l'étranger peuvent également évoluer avec le temps. À titre illustratif, la Suédoise pourrait amorcer l'interaction avec une figure stéréotypée de la Canadienne. Si la Canadienne a ensuite un comportement contraire aux stéréotypes propres aux Canadiens, la représentation privilégiée pourrait glisser vers celle du prototype individualisé.

La troisième dimension du modèle, l'axe de complexité identitaire, fait référence à la possibilité de prendre en considération plus d'une identité de l'étranger afin de bâtir la représentation de celui-ci. Plus précisément, lorsque seule l'identité nationale est considérée, la représentation de l'étranger repose sur le pôle négatif de cette troisième dimension. En reprenant l'exemple donné ci-dessus, si la Suédoise attribue à la Canadienne une identité professionnelle en plus de l'identité nationale, la représentation de celle-ci se situerait davantage vers le pôle positif de la complexité identitaire. Il faudrait également positionner chaque identité reconnue (canadienne, collègue) sur les dimensions de différenciation et de particularisation.

Enfin, compte tenu du profil de représentation adopté, l'effet sur la relation interpersonnelle différera. À titre d'exemple, une représentation de prototype individualisé, en comparaison au profil stéréotypé, impliquera une relation de type plus profond, due à la richesse d'information comprise dans la représentation, tandis qu'une relation développée à partir d'un profil stéréotypé restera superficielle.

\section{Deuxième partie : Penser les cultures et les identités dans la communication interpersonnelle}

\section{Chapitre 3 : L'influence des cultures sur la communication}

Ce troisième chapitre aborde avec rigueur une notion clé de la communication interculturelle, celle de culture. En début de chapitre, quelques critiques en lien avec cette notion sont présentées. Par exemple, sa nature polysémique est mise en avant, tout en soulignant le désaccord existant entre chercheurs quant à une définition précise et définitive.

L'auteur enchaîne alors avec la présentation d'une définition communicationnelle de la culture, soit « un ensemble cohérent de savoirs, socialement transmis et associés à un groupe social » (p. 111). Quelques précisions sont par la suite développées. D’abord, la culture serait un phénomène groupal et aurait la possibilité d'évoluer par l'entremise d'interactions entre membres d'un même groupe social. Par la suite, l'auteur souligne qu'il y aurait plusieurs cultures présentes dans une même société, toutes pouvant s'influencer mutuellement. Finalement, l'auteur expose une structuration systémique de la culture en employant la métaphore d'un iceberg. C'est-à-dire que les aspects les plus superficiels d'une culture se trouvent à la surface et demeurent visibles (par ex. les artefacts), tandis que les valeurs et les croyances de base d'une culture, invisibles, se positionnent sous le niveau de l'eau.

Le chapitre se termine par une discussion à propos de l'impact à la fois cognitif et symbolique (identitaire) que peut avoir une culture sur les comportements lors d'échanges. Chacun est socialisé tout au long de sa vie, soit par sa culture nationale (socialisation primaire), soit par d'autres cultures lors de contacts avec celles-ci (socialisation secondaire). Le processus de socialisation permet à l'individu de développer, entre autres, sa manière de réfléchir et sa manière d'interpréter le monde. C'est l'impact cognitif. Par ailleurs, lorsqu'un individu interagit avec d'autres, il peut décider de défendre une certaine culture. Cette revendication sert à démontrer son appartenance à un groupe social. C'est l'impact symbolique ou identitaire. Selon l'auteur, les cultures de socialisations jouent un rôle à la fois cognitif et identitaire lors d'une interaction.

\section{Chapitre 4 : Penser les identités dans la communication interpersonnelle}

Ayant défini rigoureusement la notion de culture dans le chapitre 3, Alexander Frame s'attaque à opérationnaliser davantage le concept d'identité, et ce, dans le but de comprendre l'influence que peuvent avoir les différentes identités de chacun sur les comportements lors d'un échange. 
Pour ce faire, l'auteur révise en détail la théorie de l'identité sociale, théorie proposant une vision structurale de I'identité (courant d'interactionnisme symbolique, voir Stryker et Burke [2000], sociologues). Essentiellement, la théorie de l'identité sociale postule que les rôles sociaux sont les fruits des structures sociales. À l'intérieur de ces structures, la façon dont l'individu définit et se représente son rôle va contribuer au développement de son identité. Bien évidemment, l'individu occupe plusieurs rôles dans la société et développera ainsi de multiples identités. Par la suite, deux autres types d'identité ont été rajoutés à l'identité de rôle, notamment l'identité sociale et l'identité de personne. Contrairement à l'identité de rôle ( $p$. ex. père) qui se fonde sur les positions qu'occupe l'individu dans la société, l'identité sociale (p. ex. Canadien) se base sur l'appartenance à des groupes sociaux et l'identité de personne (p. ex. optimiste) repose sur des traits de personnalité.

Cette théorie postule que les individus seront motivés à faire valider leurs identités. C'est donc avantageux pour l'individu que sa perception de soi concorde avec la perception que les autres ont de lui. La théorie postule que lorsqu'un individu parvient à faire valider son identité, il bénéficie d'une hausse d'estime de soi. L'estime de soi peut par contre diminuer lorsque cette validation de soi n'a pas lieu. II est à noter que l'importance accordée à l'identité en question influencera l'ampleur de la hausse ou de la baisse d'estime de soi.

Le chapitre se termine par l'exposition de certaines critiques énoncées à l'égard de cette théorie, notamment sa nature ethnocentrique. Plus précisément, le développement et la vérification de cette théorie ont eu lieu principalement aux États-Unis. La possibilité que d'autres cultures présentent des modes de fonctionnement cognitif différents de ceux des Américains est donc fort probable et reste à vérifier.

\section{Troisième partie : Penser l'interculturalité de la communication}

\section{Chapitre 5 : La négociation du sens pendant une interaction}

Le cinquième chapitre se focalise sur le processus dynamique de sémiogénèse communicationnelle, défini comme le processus « d'émergence de significations fondées sur les cultures et les identités, qui prennent forme dans un contexte interactionnel (figuratif) particulier » (p. 173). L'auteur souligne l'énorme défi qui se pose lorsqu'on tente d'analyser le processus de construction de sens pendant une interaction. Il souligne l'implication de deux processus dans la coconstruction du sens, soit la négociation identitaire et la coordination des codes.

L'auteur explique qu'afin de donner un sens à une interaction, on aura tendance à se baser sur une définition de la situation dans laquelle on se trouve. Justement, c'est par l'entremise de son identité que l'individu donnera un sens à une situation, raison pour laquelle l'auteur nous présente d'abord le processus cognitif de négociation identitaire.

Les individus impliqués dans une interaction seront motivés à négocier leurs identités. Une négociation réussie de son identité implique que l'identité défendue concorde avec l'identité que nous attribue l'interlocuteur. Lorsque ceci se produit, le philosophe McCall et le sociologue Simmons (1978) parlent de " modus vivendi ». C'est en partie grâce à un « modus vivendi » bien établi que l'individu saura donner sens à l'interaction.

En plus de négocier leurs identités, et afin de structurer le sens d'une interaction, les individus devront également coordonner leurs codes en entreprenant un processus de coconstruction de sens. Le processus de coconstruction de sens se base sur des inférences individuelles qui, lorsqu'elles sont jumelées à celles de l'interlocuteur, permettent la mise en place d'un accord temporaire de la définition de la situation.

La coconstruction de sens est un processus complexe qui se fonde sur des automatismes cognitifs (Gudykunst, 1995). L'auteur présente par exemple le processus cognitif d'inférence conversationnelle, qui implique que l'individu s'appuie sur des indices contextuels ( $p$. ex. le ton de voix), définis comme étant des "signes métalinguistiques verbaux et non verbaux qui servent à signaler les présuppositions liées au contexte " (Gumperz, 1996, p. 379), afin de donner sens à la situation. La coconstruction du sens peut s'avérer plus difficile lors d'interactions interculturelles, puisque les individus impliqués ne partagent pas le même implicite culturel. C'est lors de telles situations que les indices contextuels seront modifiés consciemment ( $p$. ex. vocabulaire simplifié).

Alterstice - Revue Internationale de la Recherche Interculturelle, vol. 3, $n^{\circ} 2$ 
Un deuxième exemple de processus cognitif automatique est la négociation d'une vision d'un monde idéal (esthétique de la situation) qui a trait à la situation particulière des individus en interaction. En d'autres termes, les individus impliqués dans l'interaction imagineront et construiront ensemble une vision idéale du monde. Ce monde imaginé contient des éléments en lien avec certains comportements prescrits et associés à certaines figures particulières. L'auteur donne l'exemple d'un couple amoureux qui élabore son monde possible. Ce monde engloberait les figures d'un jeune homme amoureux et d'une jeune femme amoureuse ainsi que quelques activités prescrites à la figure des jeunes amoureux (p. ex. sortir au cinéma). Les acteurs seront alors motivés à se comporter conformément à cette vision afin de bénéficier d'un sentiment d'authenticité par rapport à leur identité en lien avec la situation. En bref, le processus de négociation de sens lors d'interactions est un processus complexe qui implique des individus en tant qu'agents actifs.

Enfin, le chapitre se termine avec le constat que l'approche sémiopragmatique s'appuie sur une perspective diachronique de l'interaction, afin de faire sens de la communication interculturelle. Contrairement à la perspective synchronique, qui s'intéresse, elle, à un phénomène précis dans le temps sans s'intéresser à son évolution, la perspective diachronique se fonde sur l'évolution de la négociation identitaire et la coconstruction des sens à travers le temps pour expliquer en retour l'évolution de la relation interpersonnelle. Ce constat permet de postuler que la prévisibilité d'une interaction croît au fil du temps car l'ampleur des comportements envisagés comme possibles par l'interlocuteur diminue.

\section{Chapitre 6 : Interculturalité et interculturation}

Ce sixième et dernier chapitre aborde une discussion concernant l'interculturation et l'interculturalité. L'interculturation fait référence à " la manière dont les cultures coexistent et coévoluent à l'intérieur d'une société, entre sociétés, et dans le monde entier " (p. 248), tandis que l'interculturalité fait référence à "l'ensemble de processus impliqués par l'interaction entre plusieurs cultures ou individus multiculturels » (p. 287).

S'appuyant sur sa définition élargie de la culture, Frame postule que toute communication interpersonnelle comprend une dimension d'interculturalité. Deux continuums sont présentés, le premier faisant référence à une lecture "objective" et le second à une lecture "subjective" de l'interculturalité lors d'une interaction. Ces continuums mettent terme à la dichotomie arbitraire, basée sur la culture nationale, utilisée auparavant pour déterminer quelles interactions qualifier d'interculturelles.

L'auteur commence d'abord par la présentation d'un continuum d'interculturalité qui permet de " différencier les interactions selon leur degré d'altérité culturelle»(p.250). À un extrême du continuum, on retrouve les communications entre individus de cultures très similaires (communication " ordinaire ») et, à l'autre extrême, on retrouve les communications entre individus de cultures très éloignées. Ce premier continuum se veut « objectif ». L'auteur précise ici le sens du terme : "objectif " s'oppose à " subjectif » et ne renvoie pas au sens d'une fiabilité de la mesure. Par exemple, un chercheur aura recours à ce continuum pour évaluer la différence " objective " entre deux cultures, et ce, dans le but de déterminer leur degré de proximité ou d'éloignement sur le continuum. Ainsi, une communication entre deux individus appartenant à la même culture nationale sera qualifiée de " communication ordinaire ", tandis qu'une interaction impliquant des individus de différentes nationalités, malgré leur appartenance partagée à d'autres cultures ( $p$. ex. professionnelle), sera plutôt considérée comme « interculturelle».

L'auteur poursuit en présentant un second continuum d'interculturalité, qui prend en compte les représentations intersubjectives des individus impliqués dans l'interaction. Plus précisément, ce continuum représente une mesure subjective du degré d'éloignement entre les deux cultures en contact. Ce degré d'éloignement subjectif réfère aux quatre modalités de représentations de l'étranger présentées dans la première partie. Par exemple, si l'individu perçoit l'étranger comme appartenant à une culture similaire à la sienne (communication "ordinaire »), il le représentera en tant que sosie culturel.

L'auteur soulève qu'il serait fort intéressant non seulement d'étudier les communications interculturelles entre cultures éloignées, mais aussi d'explorer davantage les communications interculturelles entre membres de cultures 
similaires. À titre d'exemple, l'auteur cite sa thèse de doctorat, qui portait sur l'analyse microsociale (par opposation à l'analyse macrosociale, qui s'intéresse aux cultures nationales) des interactions entre les membres d'une association étudiante européenne, et ce, entre autres, dans le but d'identifier les traits culturels mis en avant lors de la communication (Frame, 2008).

Ce dernier chapitre se conclut par la présentation du processus d'interculturation (défini ci-dessus). L'auteur souligne d'abord l'importance des interactions sociales dans l'évolution des cultures. D'ailleurs, quelques types d'évolution de la culture sont présentés, dont certains sont conscients et d'autres inconscients (p. ex. l'emprunt et l'innovation). L'avantage majeur du modèle sémiopragmatique serait sa capacité à permettre la conceptualisation du lien entre le micro et le macro. Contrairement aux nombreuses études et théories interculturelles, le modèle sémiopragmatique ne postule pas qu'il existe un lien direct entre "les microprocessus communicationnels et l'évolution des cultures macrosociologiques » (p. 259). Au contraire, ce modèle souligne l'importance du contexte et de la situation sur le lien entre le micro et le macro.

Enfin, l'auteur illustre à l'aide d'une figure les différents liens possibles entre groupes sociaux. Cette figure parvient à synthétiser les trois types de rapports existants entre groupes, soit les rapports hiérarchiques intra-sociétaux, les rapports de transversalité intra-sociétale et les rapports de transversalité inter-sociétale. Trois groupes sociétaux sont illustrés dans la figure (groupe A, B et C), ainsi que les groupes intra-sociétaux correspondants (groupes A1, A2, B1, B2, C1 et C2). Dans le but d'illustrer le fonctionnement de la figure, attribuons au groupe social A la culture nationale canadienne, au groupe B, la culture nationale suédoise, aux groupes A1 et B1 le groupe social des femmes et aux groupes $A 2$ et B2 le groupe social des hommes. Le lien entre le groupe canadien (A) et celui des femmes (A1) ou des hommes (A2) canadiens est qualifié de rapport hiérarchique intra-sociétal, tandis que le lien unissant le groupe des femmes (A1) et des hommes (A2) canadiens entre eux réfère au rapport de transversalité intra-sociétale. Le dernier lien illustré est celui des rapports de transversalité inter-sociétale, soit le lien entre les femmes canadiennes (A1) et les femmes suédoises (B1).

Cette figure contribue à la compréhension du phénomène d'interculturation, en permettant de penser les liens entre différents groupes sociaux en termes de recoupements, recouvrements et englobements plutôt que d'effectuer simplement une distinction entre cultures microsociales et macrosociales. En allant au-delà des distinctions macro- et micro-sociales, cette figure permet de penser les cultures transnationales (p. ex. les jeunes) qui ne peuvent être qualifiées ni de microsociales ni de macrosociales.

\section{Conclusion}

En conclusion, Frame examine les multiples champs de recherche pouvant bénéficier d'applications pratiques de l'approche sémiopragmatique. À titre illustratif, la sociologie de la famille et le domaine de la communication organisationnelle pourraient s'appuyer sur ce modèle pour étudier les répercussions que les cultures familiales ou organisationnelles peuvent avoir sur les interactions. Par ailleurs, une nouveauté de ce modèle est le succès avec lequel il parvient à prendre en considération l'influence de multiples cultures et identités sur la communication interculturelle. Enfin, en restant réaliste, l'auteur estime qu'il reste à vérifier si ce modèle parvient à déjouer l'ethnocentrisme.

\section{Commentaires}

En bref, l'objectif de l'auteur était de développer un cadre théorique innovateur permettant de conceptualiser la communication interpersonnelle (rappelons que toutes les interactions interpersonnelles comprennent un certain degré d'interculturalité selon Frame), et ce, en accordant une grande importance au rôle que les cultures et les identités peuvent jouer sur la communication. L'auteur parvient avec succès à atteindre cet objectif. II présente de façon rigoureuse, systématique et critique les théories préexistantes qui ont trait à la communication interculturelle, tout en apportant des nouveautés à celles-ci afin d'en combler les lacunes. L'insertion d'exemples concrets et de figures rend la lecture plus facile et permet au lecteur de bien saisir les théories présentées. Par ailleurs, le regard multidisciplinaire que Frame privilégie assure à l'approche sémiopragmatique une contribution provenant d'une variété de domaines d'expertise. Frame cite ainsi des auteurs issus entre autres de la psychologie, la sociologie, la communication, l'anthropologie et la philosophie.

Alterstice - Revue Internationale de la Recherche Interculturelle, vol. $3, n^{\circ} 2$ 
De plus, cet ouvrage vient combler une lacune importante de la recherche interculturelle, qui est celle de cibler presque uniquement la communication entre individus de cultures nationales différentes. En proposant d'élargir nos horizons et de constater que toute interaction interpersonnelle comprend une dimension d'interculturalité, Frame apporte une contribution importante au domaine de la communication interculturelle.

Cette contribution ouvre, en retour, une porte à de nombreuses pistes de recherche encore inexplorées, et ce, dans plusieurs disciplines (communication, psychologie, sociologie, etc.). Par exemple, on pourrait se pencher sur les identités privilégiées lors d'interactions entre individus appartenant à une même culture nationale mais différant quant à leur appartenance à un groupe social.

Cet ouvrage est à recommander à tous chercheurs, théoriciens et professionnels s'intéressant à des problématiques touchant à la culture, à l'identité, à la communication ou à l'interculturalité. C'est en retravaillant certains concepts de base avec lesquels nombre d'entre nous travaillons quotidiemment qu'Alexander Frame nous permet de développer une nouvelle façon de se les représenter.

\section{Références bibliographiques}

Blumer, H. (1969). Symbolic interactionism: perspective and method. Berkley, CA : University of California Press.

Frame, A. (2008). Repenser l'interculturel en communication: performance culturelle et construction des identités au sein d'une association européenne (thèse de doctorat, Université de Bourgogne, France).

Frame, A. (2013). Communication et interculturalité : cultures et interactions interpersonnelles. Paris : Lavoisier.

Giles, H. (1973). Accent mobility: a model and some data. Anthropological linguistics, 15(2), 87-105.

Gudykunst, W. (1995). Anxiety/Uncertainty Management (AUM) theory: current status. Dans R. Wiseman (dir.), intercultural communication theory (p. 8-58). Thousand Oaks, CA : Sage .

Gumperz, J. (1996). The linguistic and cultural relativity of conversational inference. Dans J. Gumperz et S. Levinson, Rethinking Linguistic Relativity (p. 374-405). Cambridge : Cambridge University Press.

Hofstede, G. (1980). Culture's consequences: international differences in work-related values. Beverly Hills, CA : Sage Publications.

McCall, G., \& Simmons, J. (1978). Identities and interactions. An examination of human associations in everyday life. New York : Free Press.

Stryker, S. (1980). Symbolic interactionism: social structural version. San Francisco, CA : Benjamin/Cummings.

Stryker, S. et Burke, P. (2000). The past, present, and future of an identity theory. Social psychology quarterly, 63(4), 284-297. 